\title{
Deep Learning based Computer Aided Diagnosis System for Breast Mammograms
}

\author{
M. Arfan Jaffar \\ College of Computer and Information Sciences, \\ Al Imam Mohammad Ibn Saud Islamic University (IMSIU), \\ Riyadh, Saudi Arabia
}

\begin{abstract}
In this paper, a framework has been presented by using a combination of deep Convolutional Neural Network (CNN) with Support Vector Machine (SVM). Proposed method first perform preprocessing to resize the image so that it can be suitable for CNN and perform enhancement quality of the images can be enhanced. Deep Convolutional Neural Network (CNN) has been used for features extraction and classification with Support Vector Machine (SVM). Standard dataset MIAS and DDMS has been employed for testing the proposed framework by generating new images from these datasets by the process of augmentation. Different performance measures like Accuracy, Sensitivity, Specificity and area under the curve (AUC) has been employed as a quantitative measure and compared with state of the art existing methods. Results shows that proposed framework has attained accuracy $93.35 \%$ and 93\% sensitivity.
\end{abstract}

Keywords-Classification; breast mammograms; computer aided diagnosis; deep learning

\section{INTRODUCTION}

Cancer is the utmost precarious and foremost source of death in the entire world. It can effect different organs of human very badly and very fast. With the passage of time, it went to the stage where it is not possible to diagnose. It is possible to handle it at initial stages. Breast cancer is one of the conspicuous causes for deaths of women, and according to a survey and report published in 2016 statistics shows [1], that 61,000 new circumstances of breast cancer are prophesied. It has been well established and well reported in the literature that if breast cancer [2] is perceived at early stages by mammographic screening process then there are many chances to survive for these types of cancerous patients and even survival rate can be increased more than $90 \%$. Therefore, for analysis and identification of breast cancer, the digital screening mammography [3] is broadly employed by radiologists. Computer aided diagnosis (CAD) is a process to diagnose automatically by using digital process by using some intelligent methods. In the literature, it has been reported that CAD systems has been used successfully for different types of cancer to diagnose at initial stages [4], [5]. It is very important to diagnose initially at early stages otherwise it is very difficult to handle it. Second the radiologist is very expensive in the whole world wide. Cancer is very dangerous disease so it is very important to have different opinions from different experts. It is very difficult for common person to consult more than one radiologist. Thus CAD can be used with radiologist as a second opinion [6]. Different intelligent techniques have been used in the literature for CAD. Mostly existing methods used features extraction by extracting different types of features [7], [8]. These features include texture, statistical, shape or gradient based approaches. After extracting features, intelligent classifiers have been used. But the basic problem with these type of features is not robust. There is no standard way to check which features are most suitable for CAD and which classifier is most suitable. Thus features extraction is a big problem. To handle such type of issues, deep learning based methods has been proposed that used raw images as input [9], [10]. These deep learning approaches used raw images as input and extract and learn features directly from images. Then these features can be used for training and testing by fully connected deep layers of neural network. There are different models of deep learning are available like convolutional neural network (CNN) and deep belief network (DBN) [11], [12]. It has been presented in the literature that deep learning based methods perform well to solve pattern recognition problems specially in medical domain. Fig. 1 shows some images from DDMS dataset to show benign and malignant samples [13]. Table 1 show all abbreviation employed in this paper.

In the literature, it has been shown that in the previous decades, most of the researchers focus on some preprocessing and post-processing operation like segmentation, background removal, pectoral removal and some other methods for CAD [14]-[16]. These old techniques are now old and even required more processing time as well as some expert knowledge also required to highlight that which part is breast part and which part is not. Similarly, for post-processing some features extraction methods have been performed like texture features, some geometrical features, statistical features or some other features to classify images. Again there is no rule that which features are most suitable. Classification has been performed by using some old classifiers like artificial neural network, KNN, Bayesian [17], [18]. But most of the times, these features extraction and classifiers are not suitable. Even best features selection and classifiers selection is also a difficult task. With the concept of new advanced concept of deep learning, all these preprocessing and post-processing steps has been removed. Deep learning used original images as input, extract features automatically and learn it from direct pixels [19], [20]. It has been used in may real time applications and to solve complex problem. Basically, deep learning converts the original input images to features representation at different layers [21], [22]. These layers' work based upon some filters like edge detection filters or line detection. Most of the studies 
in literature shows that the most suitable model is deep Convolutional Neural Network (CNN) that works just like Multi-Layer Perceptron (MLP) with some modifications. So in this paper, CNN has been used with SVM to extract features automatically and then classify by using deep concepts learning. Some preprocessing has been performed to resize the images. Most of the time the images used in mammography are $1024 \times 1024$ so it is very difficult to process it directly on such huge size of the image [23]. Therefore, resizing has been applied first. Then to smooth the images while retaining the edges preserved Bilateral filter has been employed with log transformation process. It enhances the quality of the images as shown in Fig. 2 and 3. The major contribution of the proposed method is to utilize the enhancement process by using hybrid bilateral filter with log transformation. Another one is to use deep convolutional neural network for the features extraction and classification.

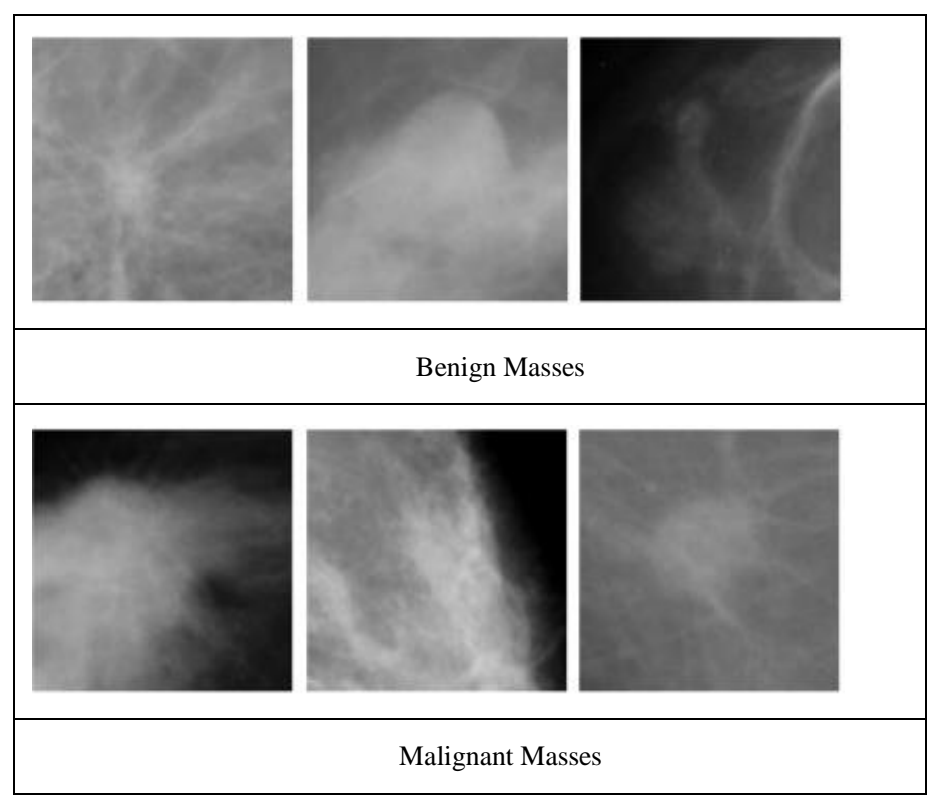

Fig. 1. Benign and Malignant Masses from DDMS dataset.

\section{PROPOSED METHOD}

Deep convolutional neural network (CNN) has been employed in the proposed framework. The proposed framework used preprocessing step to enhance the quality of the images. Fig. 1 shows sample image taken from DDMS dataset and it clearly shows that this mammogram images are not high contrast. Some images are not clearly visible and good quality due to low resolution and sometimes due to conversion. To improve the quality of the images, enhancement process is required so that it can be improved and enhanced for good quality. Enhancement also improve quality so it can be more feasible for features extraction. Then, then extracted features are required to classify those regions. Deep CNN has been used with SVM for features extraction and classification. Details of all these phases has been given below in detail

\section{A. Dataset Description}

We have used two standard datasets MIAS-mini [20] and DDMS [21] that are mostly used in the literature for breast mammograms classification and also augmented the images to generate a large dataset that is suitable for deep convolutional neural network. Augmentation process has been explained in the preprocessing step in detail. In These both datasets are supervised that mean class labels of both datasets are given with data information. From MIAS dataset 322 images and from DDMS 1800 images has been selected to use in this study. Third dataset has been created artificially by augmentation process. For data augmentation rotation has been performed on both MIAS and DDMS images by $0,45,90,135$, $180,225,270,315$ and 360 degrees to generate huge dataset.

TABLE I. LIST OF ABbREVIATION USED IN THIS PAPER

\begin{tabular}{|c|c|}
\hline Abbreviation & Complete Name \\
\hline Log Transformation & $\begin{array}{l}\text { Logarithmic } \\
\text { Transformation }\end{array}$ \\
\hline SVM & Support Vector Machine \\
\hline Max Pooling & Maximum Pooling \\
\hline $\mathrm{CNN}$ & $\begin{array}{l}\text { Convolutional Neural } \\
\text { Network }\end{array}$ \\
\hline CAD & Computer Aided Diagnosis \\
\hline MIAS & $\begin{array}{l}\text { Mammographic image } \\
\text { analysis society }\end{array}$ \\
\hline DDMS & $\begin{array}{l}\text { Digital database for } \\
\text { screening mammography }\end{array}$ \\
\hline AUC & $\begin{array}{l}\text { Area under the receiver } \\
\text { operating curve }\end{array}$ \\
\hline ReLU & Rectified Liner Unit \\
\hline
\end{tabular}

\section{B. Preprocessing}

After taking images from these datasets, it is required to enhance the visual quality of mammogram images. So a framework or procedure is required so that quality and visibility of the images can be enhanced. There are different enhancement methods available in the literature but most suitable enhancement method for mammograms is to improve the breast part in such a way that it also preserves the edges information. First, to decrease the size of mammograms, resizing has been applied so that it can be suitable for convolution neural network. So mammogram images are resized to suitable size 96x96 using a special technique bicubic interpolation. After resizing to proper size, bilateral filter employed on the images. Bilateral filter enhances the images but also preserve edges. We have tried many other methods based upon histogram but bilateral filter performs well as compare to other methods. So first original images processed to enhance the quality by incorporating bilateral filter [7]. This filter improves the image quality and make it smooth. Then logarithm transformation [8] employed on the filtered images returned by bilateral filter processed images. The resultant images displayed in Fig. 2. It improves the dynamic range especially for dark portion in the images as shown in Fig. 3. 


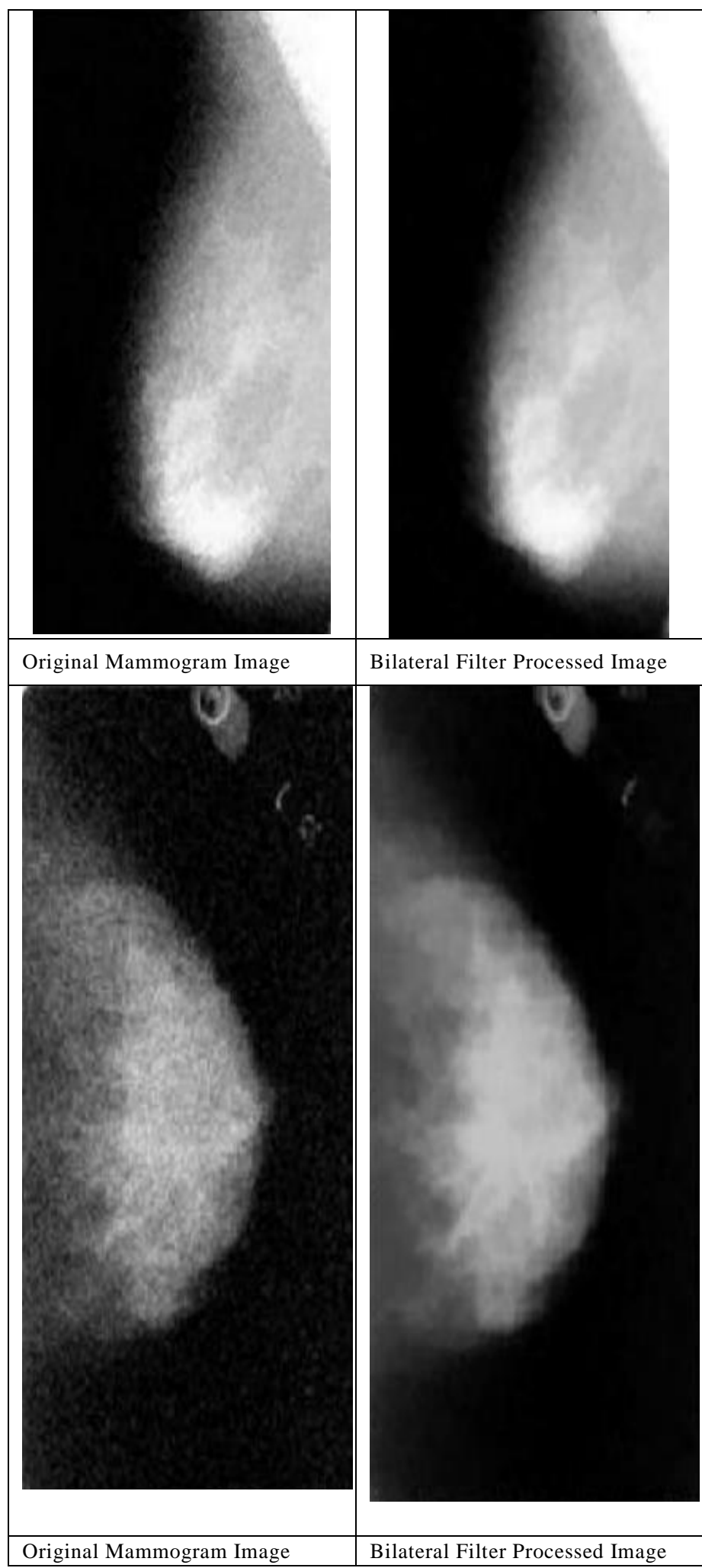

Fig. 2. Bilateral filter processed images results.

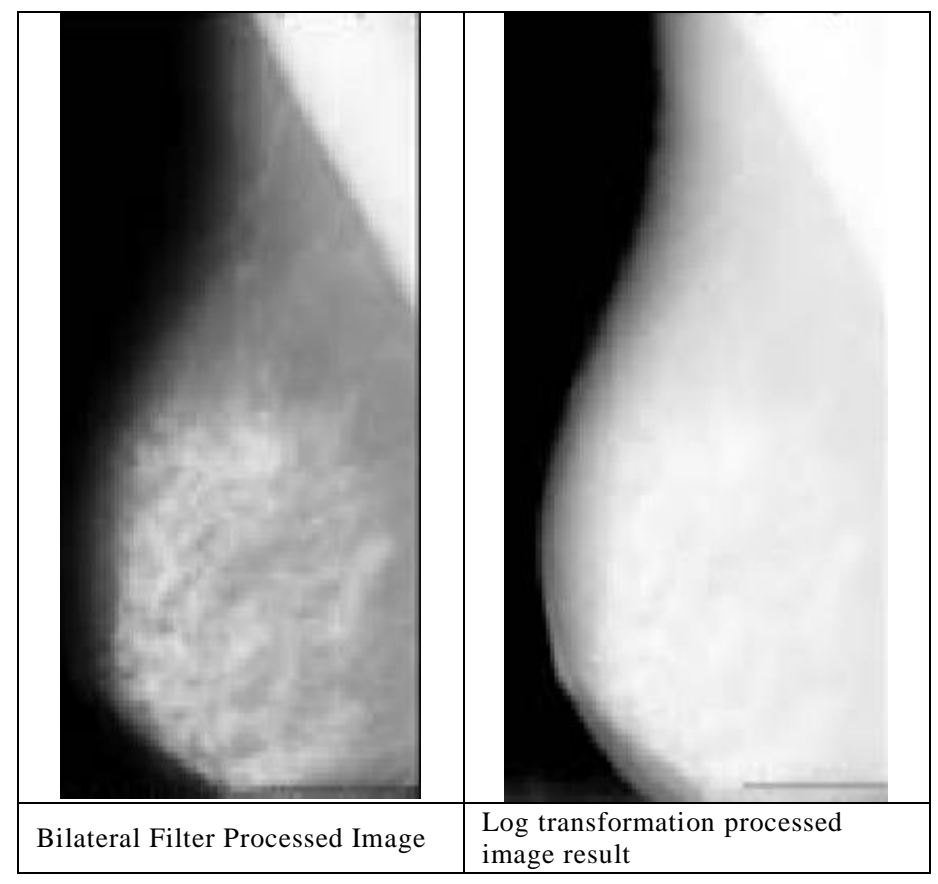

Fig. 3. Log Transformation processed images results.

Results of Bilateral filter and log transformation has been shown in Fig. 2 and 3. These images show clearly improvement by applying this process. At the end, to increase the training data, we created many images from existing data available in the datasets so that it can be suitable for convolutional neural network. For this purpose, rotation has been performed on all existing images at different angles by 0 , $45,90,135,180,225,270,315$ and 360 . So in this way, a large training dataset has been created Finally, we augmented the images to have a bigger training set. For data augmentation we rotated the images by $0,45,90,135,180,225,270,315$ and 360 degrees. After creating all these images, horizontal flip operation has been performed on all these artificially created images by rotation.

\section{Deep Convolutional Neural Network}

For classification, feed-forward convolutional neural network has been applied for the classification of mammogram images. The network used in this paper has been shown in Fig. 4. Three convolution layers has been used for experimentation. Three layers has three filters 46, 128 and 256 respectively and each filter has a size of $5 \times 5$. Rectified Liner Unit (ReLU) has been used after these filters as activation functions. After using two convolutional layers at first two layers, max pooling has been employed at the third layer and after third layer, quadrant pooling has been applied. After that, fully connected layers has been used with 400 hidden neurons and at the end softmax layer has been used for classification. Max pooling concept has been used for filtering the process at each layer as shown in Fig. 5 and this figure has been download from internet. Biases has not been used during experimentation. The major advantage of not using biases is that it increases the speed of the network so that it can be trained very efficiently and it also decreases the total amount of parameters that required learning during training [10]. 


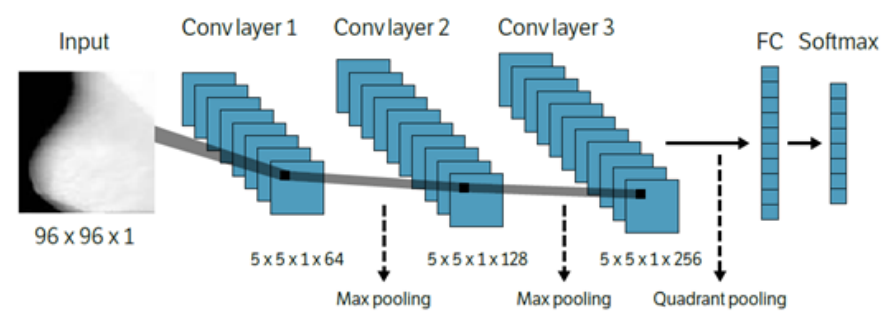

Fig. 4. Deep convolutional neural network model.
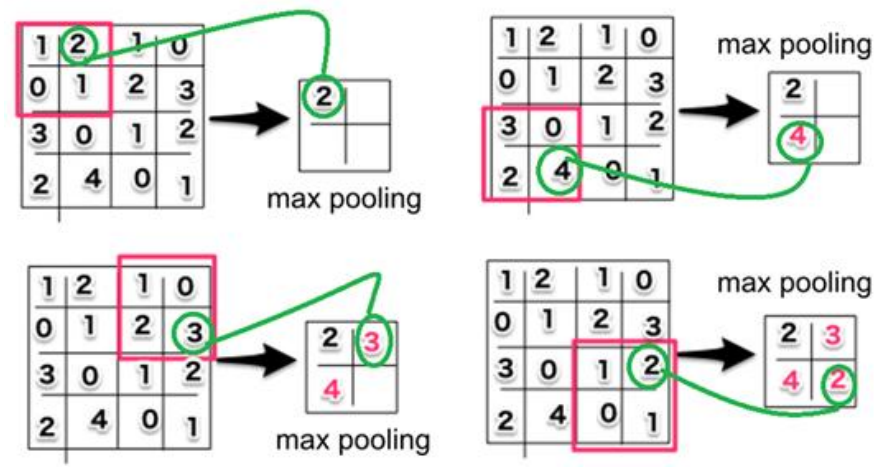

Fig. 5. Process of Max Pooling applied in CNN.

For training the network, stochastic gradient descent method has been used. Batch size for this stochastic gradient descent set to 64 with momentum 0.8 . And decay parameter set to 1e-5. Dropout mechanism also used to the fully-connected layer by setting probability to 0.5 . Training has been performed by using the model of Anna software library. After training and features extraction along with corresponding labels of mammograms, it is used to train the Support Vector Machine (SVM) classifier. Training has been performed on different classifiers for each network. Finally, at the end, average has been used to calculate the average class score of all these different classifiers for the classification result as output.

\section{RESULTS AND DISCUSSION}

Different performance measures have been used for the validation of proposed method. Classification results have been shown in the form of accuracy in Table 2. To calculate accuracy, a probability of $50 \%$ was used as threshold. For experimentation and testing, laptop with NVIDIA GTX 840M 2GB, 6 GB installed memory and Intel Core i7-4500U $1.8 \mathrm{GHz}$ CPU has been used. MATLAB 2016b version has been for implementation and MatConvNet framework has been used for CNN. It takes around 120 minutes for training and for testing; it takes only 7 minutes for all test images.

Proposed method has been tested on large number of images taken from two standard datasets MIAS (322 images) and DDMS (1800 images) and also generated artificially by augmentation process explained in section 2.1 so total approximately 19000 image dataset has been generated by using this process. To generate the training and testing data, 10 -fold cross validation process has been used with $50 \%$ training and 50\% testing. Four different measures have been used to validate the results. Different performance measures like Accuracy, Sensitivity, Specificity and area under the receiver operating curve (AUC) [23] has been employed to check the output of the proposed framework. These are the standard measures used for classification and specially AUC is the most common to assess the overall discrimination. Greater value of AUC shows good performance that should be close to 1. Proposed method has been compared with state-of-the-art methods that used the same concept like deep learning classification algorithms. The most common method used in this area are DeepCAD [17], CNN-Max-CAD-Qiu [15] and CNN-CAD-Jiao [16] by using same parameters and datasets. Table 2 shows results of the proposed method on different datasets like MIAS-mini, DDMS, combination set of MIASmini and DDMS and combination of MIAS-mini, DDMS and artificially generated datasets. This table shows the if CNN has been trained on large dataset then it also enhances the performance of the proposed method. It clearly shows that on new artificially generated dataset the accuracy is 93.72 percent that is maximum against all other datasets. Even other measures like sensitivity is 94.19 , specificity is 92.24 and AUC is 0.93. All these values shows that it performs well on big dataset that contains approximately 19000 images.

TABLE II. DifFERENT Classifiers Results Without ENHANCEMENT

\begin{tabular}{|l|l|l|l|l|}
\hline Classifier & \multicolumn{1}{|l}{$\begin{array}{l}\text { Accuracy } \\
(\%)\end{array}$} & $\begin{array}{l}\text { Sensitivity } \\
(\%)\end{array}$ & $\begin{array}{l}\text { Specificity } \\
(\%)\end{array}$ & $\begin{array}{l}\text { AU } \\
\text { C } \\
(\%)\end{array}$ \\
\hline $\begin{array}{l}\text { MIAS- } \\
\text { mini }\end{array}$ & 92.85 & 93.25 & 90.56 & 0.92 \\
\hline \begin{tabular}{l} 
DDMS \\
\hline MIAS-
\end{tabular} & 93.02 & 92.84 & 91.35 & 0.91 \\
\hline $\begin{array}{l}\text { mini + } \\
\text { DDMS }\end{array}$ & 93.35 & 93.18 & 91.04 & 0.93 \\
\hline $\begin{array}{l}\text { New } \\
\text { Artificiall } \\
\text { y } \\
\text { generated } \\
\text { dataset }\end{array}$ & 93.72 & 94.19 & 92.24 & 0.93 \\
\hline
\end{tabular}

TABLE III. COMParison 0F Proposed Method With Different EXISTING METHODS

\begin{tabular}{|l|l|l|l|}
\hline Methodology & Dataset & $\begin{array}{l}\text { Accuracy } \\
\%\end{array}$ & $\begin{array}{l}\text { AUC } \\
\%\end{array}$ \\
\hline Ball et al. [21] & DDMS & 87 & NA \\
\hline Varela et al. [22] & DDMS & 81 & NA' \\
\hline DeepCAD [17] & $\begin{array}{l}\text { DDMS + } \\
\text { MIAS }\end{array}$ & 91 & 0.91 \\
\hline $\begin{array}{l}\text { CNN-Max-CAD- } \\
\text { Qiu [15] }\end{array}$ & $\begin{array}{l}\text { DDMS + } \\
\text { MIAS }\end{array}$ & 80 & 0.76 \\
\hline CNN-CAD-Jiao [16] & $\begin{array}{l}\text { DDMS + } \\
\text { MIAS }\end{array}$ & 73 & 0.67 \\
\hline Proposed & $\begin{array}{l}\text { DDMS + } \\
\text { MIAS }\end{array}$ & 93.35 & 0.93 \\
\hline
\end{tabular}


Table 3 shows comparison of proposed method with other existing methods available in the literature. All these results for other methods has been taken reported in other research papers. The obtained results are reported in Tables 2 and 3. Table 3 results have been taken from paper [17] for DeepCAD, CNNMAX-CAD-Qiu and CNN-CAD-Jiao and for Ball and Varela has been taken from [18]. Ball shows 87\% accuracy on DDMS dataset, Varela shows $81 \%$ on DDMS dataset.

These methods have been not reported AUC in the paper therefore, I did not show results for these methods. DeepCAD has accuracy $91 \%$ and AUC 0.91 percent and author has tested on 600 ROI images collected from MIAS and DDMS. Author also shows results for other methods on the same collection of MIAS + DDMS dataset that CNN-Max-CAD-Qiu shows $80 \%$ and $0.76 \%$ accuracy and AUC accordingly and CNN-CAD-Jio shows $73 \%$ and $0.67 \%$ on the same collection of MIAS+DDMS dataset. Proposed method shows 93.35\% accuracy and $0.93 \%$ AUC on combination dataset of MIASmini + DDMS. These values shows that proposed method works better as compare to all these existing methods. The proposed system does some preprocessing to enhance the performance of images like enhancement and resizing therefore it also performs well. Second, it has been trained on large set of images generated by artificially that is also helpful for good performance.

\section{CONCLUSION}

In this paper, I have proposed a computer aided diagnosis system based upon deep convolutional neural network. There are two different tasks that has been applied. In the first task, breast preprocessing has been performed to make it suitable for CNN. During preprocessing, resizing has been performed on the images so that it can be suitable for CNN. Then dataset has been increase so that it is suitable for CNN. Different rotations have been used to generate artificially data by using the same data available in MIAS and DDMS dataset. After that, enhancement has been performed by using Bilateral filter and $\log$ transformation so that images can be smooth while preserving edges. After that CNN model has been used for features extraction classification. Results shows that it has improved the performance of CAD system by incorporating CNN. Due to these contributions, proposed system perform well. In the future, some other model of deep learning will be explored.

\section{REFERENCES}

[1] Siegel, R.L.; Miller, K.D.; Jemal, A. Cancer statistics, 2016. CA Cancer J. Clin. 2016, 66, 7-30.

[2] Kalager, M.; Zelen, M.; Langmark, F.; Adami, H.O. Effect of screening mammography on breast-cancer mortality in Norway. N. Engl. J. Med. 2010, 363, 1203-1210.

[3] Posso, M.; Carles, M.; Rué, M.; Puig, T.; Bonfill, X. Cost-Effectiveness of double reading versus single reading of mammograms in a breast cancer screening programme. PLoS ONE 2016, 11, 1-13.

[4] Bird, R.E., Professional Quality assurance for mammography screening programs, Radiology 177, pp.587. 1990.

[5] Bellotti, R., De Carlo, F., Tangaro, S., Gargano, G., Maggipinto, G., Castellano, M., ... De Nunzio, G. (2006). A completely automated CAD system for mass detection in a large mammographic database. Medical Physics, 33(8), 3066-3075.
[6] Varela, C., Tahoces, P. G., Méndez, A. J., Souto, M., \& Vidal, J. J. (2007). Computerized detection of breast masses in digitized mammograms. Computers in Biology and Medicine, 37(2), 214-226.

[7] F. Sahba, A. Venetsanopoulos, Breast mass detection using bilateral filter and mean shift based clustering, in: Proceedings of the 2010 International Conference on Signal Processing and Multimedia Applications (SIGMAP), 2010, pp. 88-94.

[8] Urvashi Manikpuri, Yojana Yadav, Image Enhancement Through Logarithmic Transformation, International Journal of Innovative Research in Advanced Engineering (IJIRAE) ISSN: 2349-2163 Volume 1 Issue 8, 2014.

[9] Wei, D., Chan, H. P., Helvie, M. a, Sahiner, B., Petrick, N., Adler, D. D., \& Goodsitt, M. M. (1995). Classification of mass and normal breast tissue on digital mammograms: multiresolution texture analysis. Medical Physics.

[10] Pooya Khorrami, Tom Le Paine, Thomas S. Huang, Do Deep Neural Networks Learn Facial Action Units When Doing Expression Recognition?, Computer Vision and Pattern Recognition, 2017 arXiv:1510.02969.

[11] T.L. Economopoulos, P.A. Asvestas, G.K. Matsopoulos, Contrast enhancement of images using Partitioned Iterated Function Systems, Image and Vision Computing Volume 28, Issue 1, January 2010, Pages 45-54.

[12] Freund Y, Schapire RE, Experiments with a new boosting algorithm. In: Machine learning: proceedings of the thirteenth international conference, 1996, pp 325-332.

[13] R. M. Haralick, K. Shanmugam, and I. Dinstein, "Textural features for image classification," IEEE Transactions on Systems, Man and Cybernetics, vol. 3, no. 6, pp. 610-621, 1973.

[14] Lior Rokach, Ensemble-based classifiers, Artificial Intelligence Reviews (2010) 33:1-39.

[15] Qiu, Y.; Wang, Y.; Yan, S.; Tan, M.; Cheng, S.; Liu, H.; Zheng, B. An initial investigation on developing a new method to predict short-term breast cancer risk based on deep learning technology. In Proceedings of the SPIE 9785, Medical Imaging 2016: Computer-Aided Diagnosis, San Diego, CA, USA, 24 March 2016.

[16] Jiao, Z.; Gao, X.; Wang, Y.; Li, J. A deep feature based framework for breast masses classification. Neurocomputing 2016, 197, 221-231.

[17] Qaisar Abbas, DeepCAD: A Computer-Aided Diagnosis System for Mammographic Masses Using Deep Invariant Features, Computers, 2016, 5, 28; doi:10.3390/computers5040028.

[18] Wentao Zhu, Qi Lou, Yeeleng Scott Vang, Xiaohui Xie, Deep Multiinstance Networks with Sparse Label Assignment for Whole Mammogram Classification, arXiv preprint arXiv:1612.05968 (2016).

[19] Suckling, J.; Parker, J.; Dance, D.; Astley, S.; Hutt, I.; Boggis, C.; Ricketts, I.; Stamatakis, E.; Cerneaz, N.; Kok, S.; et al. The mammographic image analysis society digital mammogram database. In Exerpta Medica: International Congress Series; Elsevier: Amsterdam, The Netherlands, 1994; Volume 1069, pp. 375-378. Available online: http://peipa.essex.ac.uk/info/mias.html (accessed on 28 October 2016).

[20] Heath, M.; Bowyer, K.W.; Kopans, D. The digital database for screening mammography. In Proceedings of the 5th International Workshop on Digital Mammography, Toronto, ON, Canada, 11-14 June 2000.

[21] Ball, J.E., Bruce, L.M.: Digital mammographic computer aided diagnosis (cad) using adaptive level set segmentation. In: 2007 29th Annual International Conference of the IEEE Engineering in Medicine and Biology Society. pp. 4973-4978. IEEE (2007).

[22] Varela, C., Timp, S., Karssemeijer, N.: Use of border information in the classification of mammographic masses. Physics in Medicine and Biology 51(2), 425 (2006).

[23] Metz, C.; Herman, B.; Shen, J. Maximum likelihood estimation of receiver operating characteristic (ROC) curves from continuouslydistributed data. Stat. Med. 1998, 17, 1033-1053. 